\title{
A study on Hermite-Hadamard type inequalities for s-convex functions via conformable fractional integrals
}

\author{
Erhan Set and Abdurrahman Gözpınar
}

\begin{abstract}
In the present note, firstly we established a generalization of Hermite Hadamard's inequality for s-convex functions via conformable fractional integrals which generalized Riemann-Liouville fractional integrals. Secondly, we proved new identity involving conformable fractional integrals via beta and incompleted beta functions. Then, by using this identity, some Hermite Hadamard type integral inequalities for s-convex functions in the second sense are obtained.
\end{abstract}

Mathematics Subject Classification (2010): 26A33, 26A51, 26D10, 26 D15.

Keywords: s-convex functions, Hermite-Hadamard inequality, conformable fractional integrals.

\section{Introduction}

One of the most famous inequality for convex functions is so called HermiteHadamard inequality as follows: Let $f: I \subseteq \mathbb{R} \rightarrow \mathbb{R}$ be a convex function and $a, b \in I$ with $a<b$, then

$$
f\left(\frac{a+b}{2}\right) \leq \frac{1}{b-a} \int_{a}^{b} f(x) d x \leq \frac{f(a)+f(b)}{2}
$$

This famous inequality discovered by C. Hermite and J. Hadamard is important in the literature. For more studies via Hermite Hadamard type inequalities see [13] in the references.

Definition 1.1. Let $f: I \subseteq \mathbb{R} \rightarrow \mathbb{R}$ be a function and $a, b \in I$ with $a<b$, the function $f: I \subseteq \mathbb{R} \rightarrow \mathbb{R}$ is said to be convex if the inequality

$$
f(t x+(1-t) y) \leq t f(x)+(1-t) f(y)
$$

holds for all $x, y \in I$ and $t \in[0,1]$. 
Definition 1.2. $[7,15]$ A function $f: \mathbb{R}_{+} \rightarrow \mathbb{R}$ is said to be s-convex in the second sense if

$$
f(\alpha x+\beta y) \leq \alpha^{s} f(x)+\beta^{s} f(y)
$$

for all $x, y \in \mathbb{R}_{+}$and all $\alpha, \beta \geq 0$ with $\alpha+\beta=1$.

We denote this by $K_{s}^{2}$. It is obvious that the s-convexity means just the convexity when $s=1$.

In [12] Dragomir and Fitzpatrick proved a variant of Hermite-Hadamard inequality which holds for s-convex functions in the second sense.

Theorem 1.3. Suppose that $f:[0, \infty) \rightarrow[0, \infty)$ is an s-convex function in the second sense, where $s \in(0,1]$ and let $a, b \in[0, \infty), a<b$. If $f \in L^{1}[a, b]$, then the following inequality hold:

$$
2^{s-1} f\left(\frac{a+b}{2}\right) \leq \frac{1}{b-a} \int_{a}^{b} f(x) d x \leq \frac{f(a)+f(b)}{s+1}
$$

The constant $k=\frac{1}{s+1}$ is the best possible in the second inequality in (1.2). For more study related to s-convexity in the second sense, see, e.g, (for example) ([3], [5], $[11])$.

Theory of convex functions has great importance in various fields of pure and applied sciences. It is known that theory of convex functions is closely related to theory of inequalities. Many interesting convex functions inequalities established via Riemann-Liouville fractional integrals. Now, lets us give some necessary definition and mathematical preliminaries of fractional calculus theory as follows, which are used lots of study. For more details, one can consult ([8]-[10], [14], [16]-[23], [28]).

Definition 1.4. Let $f \in L_{1}[a, b]$. The Riemann-Liouville integrals $J_{a+}^{\alpha} f$ and $J_{b-}^{\alpha} f$ of order $\alpha>0$ with $a \geq 0$ are defined by

$$
J_{a+}^{\alpha} f(x)=\frac{1}{\Gamma(\alpha)} \int_{a}^{x}(x-t)^{\alpha-1} f(t) d t, \quad x>a
$$

and

$$
J_{b-}^{\alpha} f(x)=\frac{1}{\Gamma(\alpha)} \int_{x}^{b}(t-x)^{\alpha-1} f(t) d t, \quad x<b
$$

respectively. Here $\Gamma(t)$ is the Gamma function and its definition is

$$
\Gamma(t)=\int_{0}^{\infty} e^{-x} x^{t-1} d x .
$$

It is to be noted that $J_{a+}^{0} f(x)=J_{b-}^{0} f(x)=f(x)$ and in the case of $\alpha=1$, the fractional integral reduces to the classical integral.

The beta function defined as follows:

$$
B(a, b)=\frac{\Gamma(a) \Gamma(b)}{\Gamma(a+b)}=\int_{0}^{1} t^{a-1}(1-t)^{b-1} d t, \quad a, b>0
$$


where $\Gamma(\alpha)$ is Gamma function. The incomplete beta function is defined by

$$
B_{x}(a, b)=\int_{0}^{x} t^{a-1}(1-t)^{b-1} d t, 0 \leq x \leq 1 .
$$

For $x=1$, the incomplete beta function coincides with the complete beta function. For easy understanding the computation in our theorems, let us give some properties of beta and incompleted beta function:

$$
\begin{aligned}
& B(a, b)=B_{t}(a, b)+B_{1-t}(b, a), \text { i.e } B(a, b)=B_{\frac{1}{2}}(a, b)+B_{\frac{1}{2}}(b, a) \\
& B_{x}(a+1, b)=\frac{a B_{x}(a, b)-(x)^{a}(1-x)^{b}}{a+b} \\
& B_{x}(a, b+1)=\frac{b B_{x}(a, b)+(x)^{a}(1-x)^{b}}{a+b} \\
& B(a, b+1)+B(a+1, b)=B(a, b)
\end{aligned}
$$

In [21] Sarıkaya et al. gave a remarkable integral inequality of Hermite-Hadamard type involving Riemann-Liouville fractional integrals as follows:

Theorem 1.5. Let $f:[a, b] \rightarrow \mathbb{R}$ be a positive function with $0 \leq a<b$ and $f \in L^{1}[a, b]$. If $f$ is convex function on $[a, b]$, then the following inequality for fractional integrals hold:

$$
f\left(\frac{a+b}{2}\right) \leq \frac{\Gamma(\alpha+1)}{2(b-a)^{\alpha}}\left[\left(J_{a^{+}}^{\alpha} f\right)(b)+\left(J_{b^{-}}^{\alpha} f\right)(a)\right] \leq \frac{f(a)+f(b)}{2}
$$

It is obviously seen that, if we take $\alpha=1$ in Theorem 1.5 , then the inequality (1.3) reduces to well known Hermite-Hadamard inequality as (1.1).

Hermite-Hadamard type inequalities for s-convex functions via RiemannLiouville fractional integral is given in [22] as follows:

Theorem 1.6. Let $f:[a, b] \rightarrow \mathbb{R}$ be a positive function with $0 \leq a<b$ and $f \in L_{1}[a, b]$. If $f$ is s-convex mapping in the second sense on $[a, b]$, then the following inequality for fractional integral with $\alpha>0$ and $s \in(0,1]$ hold:

$$
\begin{aligned}
2^{s-1} f\left(\frac{a+b}{2}\right) & \leq \frac{\Gamma(\alpha+1)}{2(b-a)^{\alpha}}\left[\left(J_{a^{+}}^{\alpha} f\right)(b)+\left(J_{b^{-}}^{\alpha} f\right)(a)\right] \\
& \leq \alpha\left[\frac{1}{\alpha+s}+B(\alpha, s+1)\right] \frac{f(a)+f(b)}{2}
\end{aligned}
$$

where $B(a, b)$ is Euler beta function.

Sarikaya et al. established an identity which we will generalize for conformable fractional integral in section 3 for differentiable convex mappings via RiemannLiouville fractional integral. Then they gave some results by using this identity. 
Lemma 1.7. [21] Let $f:[a, b] \rightarrow \mathbb{R}$ be a differentiable mapping on $(a, b)$ with $a<b$. If $f^{\prime} \in L[a, b]$, then the following equality for fractional integrals holds:

$$
\begin{aligned}
& \frac{f(a)+f(b)}{2}-\frac{\Gamma(\alpha+1)}{2(b-a)^{\alpha}}\left[J_{a^{+}}^{\alpha} f(b)+J_{b_{-}}^{\alpha} f(a)\right] \\
& =\frac{b-a}{2} \int_{0}^{1}\left[(1-t)^{\alpha}-t^{\alpha}\right] f^{\prime}(t a+(1-t) b) d t .
\end{aligned}
$$

Theorem 1.8. [21] Let $f:[a, b] \rightarrow \mathbb{R}$ be a differentiable mapping on $(a, b)$ with $a<b$. If $f^{\prime} \in L[a, b]$, then the following inequality for fractional integrals holds:

$$
\begin{aligned}
& \left|\frac{f(a)+f(b)}{2}-\frac{\Gamma(\alpha+1)}{2(b-a)^{\alpha}}\left[J_{a^{+}}^{\alpha} f(b)+I_{b_{-}}^{\alpha} f(a)\right]\right| \\
& \leq \frac{b-a}{2(\alpha+1)}\left(1-\frac{1}{2^{\alpha}}\right)\left|f^{\prime}(a)\right|+\left|f^{\prime}(b)\right|
\end{aligned}
$$

Recently, some authors started to study on conformable fractional integral. In [18], Khalil et al. defined the fractional integral of order $0<\alpha \leq 1$ only. In [1], Abdeljawad gave the definition of left and right conformable fractional integrals of any order $\alpha>0$.

Definition 1.9. Let $\alpha \in(n, n+1]$ and set $\beta=\alpha-n$ then the left conformable fractional integral starting at $a$ if order $\alpha$ is defined by

$$
\left(I_{\alpha}^{a} f\right)(t)=\frac{1}{n !} \int_{a}^{t}(t-x)^{n}(x-a)^{\beta-1} f(x) d x
$$

Analogously, the right conformable fractional integral is defined by

$$
\left({ }^{b} I_{\alpha} f\right)(t)=\frac{1}{n !} \int_{t}^{b}(x-t)^{n}(b-x)^{\beta-1} f(x) d x .
$$

Notice that if $\alpha=n+1$ then $\beta=\alpha-n=n+1-n=1$ where $n=0,1,2,3 \ldots$ and hence $\left(I_{\alpha}^{a} f\right)(t)=\left(J_{n+1}^{a} f\right)(t)$.

In [24] Set et.al. gave Hermite-Hadamard inequality for conformable fractional integral as follows:

Theorem 1.10. Let $f:[a, b] \rightarrow \mathbb{R}$ be a function with $0 \leq a<b$ and $f \in L_{1}[a, b]$. If $f$ is a convex function on $[a, b]$, then the following inequalities for conformable fractional integrals hold:

$$
f\left(\frac{a+b}{2}\right) \leq \frac{\Gamma(\alpha+1)}{2(b-a)^{\alpha} \Gamma(\alpha-n)}\left[\left(I_{\alpha}^{a} f\right)(b)+\left({ }^{b} I_{\alpha} f\right)(a)\right] \leq \frac{f(a)+f(b)}{2}
$$

with $\alpha \in(n, n+1]$, where $\Gamma$ is Euler Gamma function.

For some studies on conformable fractional integral, see ([1], [2], [4], [6]). In papers ([25]-[27]), Set et.al obtained some Hermite-Hadamard, Ostrowski, Chebyshev, Fejer type inequalities by using conformable fractional integrals for various classes of functions. The aim of this study is to establish new Hermite-Hadamard inequalities related to other fractional integral inequalities for conformable fractional integral. 


\section{Hermite-Hadamard's inequalities for conformable fractional integrals}

In this section, using the given properties of conformable fractional integrals, we will establish a generalization of Hermite-Hadamard type inequalities for s-convex functions. We will also noticed the relation with fractional and classical HermiteHadamard type integral inequalities.

Theorem 2.1. Let $f:[a, b] \rightarrow \mathbb{R}$ be a function with $0 \leq a<b, s \in(0,1]$ and $f \in L_{1}[a, b]$. If $f$ is an s-convex function on $[a, b]$, then the following inequalities for conformable fractional integrals hold:

$$
\begin{aligned}
& \frac{\Gamma(\alpha-n)}{\Gamma(\alpha+1)} f\left(\frac{a+b}{2}\right) \\
\leq & \frac{1}{(b-a)^{\alpha} 2^{s}}\left[\left(I_{\alpha}^{a} f\right)(b)+\left({ }^{b} I_{\alpha} f\right)(a)\right] \\
\leq & {\left[\frac{B(n+s+1, \alpha-n)+B(n+1, \alpha-n+s)}{n !}\right] \frac{f(a)+f(b)}{2^{s}} }
\end{aligned}
$$

with $\alpha \in(n, n+1], n=0,1,2, \ldots$ where $\Gamma$ is Euler Gamma function and $B(a, b)$ is a beta function.

Proof. Let $x, y \in[a, b]$. If $f$ is a s-convex function on $[\mathrm{a}, \mathrm{b}]$,

$$
f\left(\frac{x+y}{2}\right) \leq\left(\frac{1}{2}\right)^{s} f(x)+\left(\frac{1}{2}\right)^{s} f(y)
$$

if we change the variables with $x=t a+(1-t) b, y=(1-t) a+t b$,

$$
2^{s} f\left(\frac{a+b}{2}\right) \leq f(t a+(1-t) b)+f((1-t) a+t b) .
$$

Multiplying both sides of above inequality with $\frac{1}{n !} t^{n}(1-t)^{\alpha-n-1}$ and integrating the resulting inequality with respect to $t$ over $[0,1]$, we get

$$
\begin{aligned}
& \frac{2^{s}}{n !} f\left(\frac{a+b}{2}\right) \int_{0}^{1} t^{n}(1-t)^{\alpha-n-1} d t \\
\leq & \frac{1}{n !} \int_{0}^{1} t^{n}(1-t)^{\alpha-n-1} f(t a+(1-t) b) d t \\
& +\frac{1}{n !} \int_{0}^{1} t^{n}(1-t)^{\alpha-n-1} f((1-t) a+t b) d t \\
= & \frac{1}{n !} \int_{a}^{b}\left(\frac{b-x}{b-a}\right)^{n}\left(\frac{x-a}{b-a}\right)^{\alpha-n-1} f(x) \frac{d x}{b-a} \\
& +\frac{1}{n !} \int_{a}^{b}\left(\frac{y-a}{b-a}\right)^{n}\left(\frac{b-y}{b-a}\right)^{\alpha-n-1} f(y) \frac{d y}{b-a} \\
= & \frac{1}{(b-a)^{\alpha}}\left[I_{\alpha}^{a} f(b)+{ }^{b} I_{\alpha} f(a)\right] .
\end{aligned}
$$


Note that

$$
f\left(\frac{a+b}{2}\right) \leq \frac{\Gamma(\alpha+1)}{2^{s}(b-a)^{\alpha} \Gamma(\alpha-n)}\left[I_{\alpha}^{a} f(b)+{ }^{b} I_{\alpha} f(a)\right]
$$

where

$$
\int_{0}^{1} t^{n}(1-t)^{\alpha-n-1} d t=B(n+1, \alpha-n)=\frac{\Gamma(n+1) \Gamma(\alpha-n)}{\Gamma(\alpha+1)}
$$

which means that the left side of $(2.1)$ is proved. Since $f$ is s-convex in the second sense, to prove the right side of (2.1) we have the following inequalities:

$$
\begin{aligned}
& f(t a+(1-t) b) \leq t^{s} f(a)+(1-t)^{s} f(b) \\
& f((1-t) a+t b) \leq(1-t)^{s} f(a)+t^{s} f(b) .
\end{aligned}
$$

Adding these two inequalities, we get

$$
f(t a+(1-t) b)+f((1-t) a+t b) \leq\left[t^{s}+(1-t)^{s}\right][f(a)+f(b)] .
$$

Multiplying both sides of the resulting inequality with $\frac{1}{n !} t^{n}(1-t)^{\alpha-n-1}$ and integrating with respect to $t$ over $[0,1]$, we have

$$
\begin{aligned}
& \frac{1}{(b-a)^{\alpha}}\left[I_{\alpha}^{a} f(b)+{ }^{b} I_{\alpha} f(a)\right] \\
\leq & \frac{1}{n !} \int_{0}^{1} t^{n}(1-t)^{\alpha-n-1}\left[t^{s}+(1-t)^{s}\right][f(a)+f(b)] d t \\
= & \frac{1}{n !}[B(n+s+1, \alpha-n)+B(n+1, \alpha-n+s)][f(a)+f(b)] .
\end{aligned}
$$

Combining (2.3) and (2.4) completes the proof.

Remark 2.2. If we choose $s=1$ in Theorem (2.1), by using relation between $\Gamma$ and $B$ functions, the inequality (2.1) reduced to inequality (1.7).

Remark 2.3. If we choose $\alpha=n+1$ in Theorem 2.1, the inequality (2.2) reduced to inequality (1.4). And also if we choose $\alpha, s=1$ in the inequality (2.2), then we get well-known Hermite-Hadamard inequality as (1.2).

\section{Some new Hermite Hadamard type inequalities via conformable integration}

In order to achieve our aim, we will give an important identity for differentiable functions involving conformable fractional integrals as follows:

Lemma 3.1. Let $f:[a, b] \rightarrow \mathbb{R}$ be a differentiable mapping on $(a, b)$ with $a<b$. If $f^{\prime} \in L[a, b]$, then the following inequality for conformable fractional integrals holds:

$$
\begin{aligned}
& B(n+1, \alpha-n)\left(\frac{f(a)+f(b)}{2}\right)-\frac{n !}{2(b-a)^{\alpha}}\left[I_{\alpha}^{a} f(b)+{ }^{b} I_{\alpha} f(a)\right] \\
& =\frac{(b-a)}{2}\left\{\int_{0}^{1}\left[B_{1-t}(n+1, \alpha-n)-B_{t}(n+1, \alpha-n)\right] f^{\prime}(t a+(1-t) b) d t\right\}
\end{aligned}
$$


where $B(a, b), B_{t}(a, b)$ is Euler beta and incompleted beta functions respectively and $\alpha \in(n, n+1], n=0,1,2, \ldots$

Proof. Let

$$
I=\int_{0}^{1}\left[B_{1-t}(n+1, \alpha-n)-B_{t}(n+1, \alpha-n)\right] f^{\prime}(t a+(1-t) b) d t .
$$

Then, integrating by parts and changing variables with $x=t a+(1-t) b$, we can write

$$
\begin{aligned}
& I_{1}=\int_{0}^{1} B_{1-t}(n+1, \alpha-n) f^{\prime}(t a+(1-t) b) d t \\
& =\int_{0}^{1}\left(\int_{0}^{1-t} x^{n}(1-x)^{\alpha-n-1} d x\right) f^{\prime}(t a+(1-t) b) d t \\
& =\left.\left(\int_{0}^{1-t} x^{n}(1-x)^{\alpha-n-1} d x\right) \frac{f(t a+(1-t) b) d t}{a-b}\right|_{0} ^{1} \\
& +\int_{0}^{1}(1-t)^{n} t^{\alpha-n-1} f(t a+(1-t) b) \frac{d t}{a-b} \\
& =\left(\int_{0}^{1} x^{n}(1-x)^{\alpha-n-1} d x\right) \frac{f(b)}{b-a} \\
& +\frac{1}{b-a} \int_{a}^{b}\left(\frac{x-a}{b-a}\right)^{n}\left(\frac{b-x}{b-a}\right)^{\alpha-n-1} f(x) \frac{d x}{a-b} \\
& =B(n+1, \alpha-n) \frac{f(b)}{b-a}-\frac{n !}{(b-a)^{\alpha+1}}\left({ }^{b} I_{\alpha} f\right)(a) \\
& I_{2}=\int_{0}^{1} B_{t}(n+1, \alpha-n) f^{\prime}(t a+(1-t) b) d t \\
& =\left.B_{t}(n+1, \alpha-n) \frac{f(t a+(1-t) b)}{a-b}\right|_{0} ^{1} \\
& -\int_{0}^{1} t^{n}(1-t)^{\alpha-n-1} f(t a+(1-t) b) \frac{d t}{a-b} \\
& =-B(n+1, \alpha-n) \frac{f(a)}{b-a}+\frac{1}{b-a} \int_{a}^{b}\left(\frac{b-x}{b-a}\right)^{n}\left(\frac{x-a}{b-a}\right)^{\alpha-n-1} f(x) \frac{d x}{b-a} \\
& =-B(n+1, \alpha-n) \frac{f(a)}{b-a}+\frac{n !}{(b-a)^{\alpha+1}}\left(I_{\alpha}^{a} f\right)(b) \text {. }
\end{aligned}
$$

It means that $I=I_{1}-I_{2}$. Thus, by multiplying both sides by $\frac{b-a}{2}$ i.e

$$
\frac{b-a}{2} I=\frac{b-a}{2} I_{1}-\frac{b-a}{2} I_{2}
$$

we have desired result.

Remark 3.2. If we choose $\alpha=n+1$ in Lemma 3.1, the equality (3.1) becomes the equality (1.5). 
Now, using the obtained identity, we will establish some inequalities connected with the left part of the inequality (2.1)

Theorem 3.3. Let $f:[a, b] \rightarrow \mathbb{R}$ be a differentiable mapping on $(a, b)$ with $a<b$. If $f^{\prime} \in L[a, b]$ and $\left|f^{\prime}\right|$ is s-convex in the second sence with $s \in(0,1]$, then the following inequality for conformable fractional integrals holds:

$$
\begin{aligned}
& \left|B(n+1, \alpha-n)\left(\frac{f(a)+f(b)}{2}\right)-\frac{n !}{2(b-a)^{\alpha}}\left[I_{\alpha}^{a} f(b)+{ }^{b} I_{\alpha} f(a)\right]\right| \\
\leq & \frac{b-a}{2}\left[\frac{\left|f^{\prime}(a)\right|+\left|f^{\prime}(b)\right|}{s+1}\right] \\
& \left\{B_{\frac{1}{2}}(\alpha-n+s+1, n+1)-B_{\frac{1}{2}}(n+1, \alpha-n+s+1)\right. \\
& \left.+B_{\frac{1}{2}}(n+s+2, \alpha-n)-B_{\frac{1}{2}}(\alpha-n, n+s+2)+B(n+1, \alpha-n)\right\}
\end{aligned}
$$

where $B(a, b), B_{t}(a, b)$ is Euler beta and incompleted beta functions respectively and $\alpha \in(n, n+1], n=0,1,2, \ldots$

Proof. Taking modulus on Lemma 3.1 and using s-convexity of $\left|f^{\prime}\right|$ we get:

$$
\begin{aligned}
& \left|B(n+1, \alpha-n)\left(\frac{f(a)+f(b)}{2}\right)-\frac{n !}{2(b-a)^{\alpha}}\left[I_{\alpha}^{a} f(b)+{ }^{b} I_{\alpha} f(a)\right]\right| \\
= & \frac{b-a}{2}\left|\int_{0}^{1}\left[B_{1-t}(n+1, \alpha-n)-B_{t}(n+1, \alpha-n)\right] f^{\prime}(t a+(1-t) b) d t\right| \\
\leq & \frac{b-a}{2} \int_{0}^{1}\left|\left[B_{1-t}(n+1, \alpha-n)-B_{t}(n+1, \alpha-n)\right]\right|\left|f^{\prime}(t a+(1-t) b)\right| d t \\
= & \frac{b-a}{2} \int_{0}^{\frac{1}{2}}\left[B_{1-t}(n+1, \alpha-n)-B_{t}(n+1, \alpha-n)\right]\left|f^{\prime}(t a+(1-t) b)\right| d t \\
& +\int_{\frac{1}{2}}^{1}\left[B_{t}(n+1, \alpha-n)-B_{1-t}(n+1, \alpha-n)\right]\left|f^{\prime}(t a+(1-t) b)\right| d t \\
\leq & \frac{b-a}{2}\left\{\int_{0}^{\frac{1}{2}} B_{1-t}(n+1, \alpha-n)\left(t^{s}\left|f^{\prime}(a)\right|+(1-t)^{s}\left|f^{\prime}(b)\right|\right) d t\right. \\
& -\int_{0}^{\frac{1}{2}} B_{t}(n+1, \alpha-n)\left(t^{s}\left|f^{\prime}(a)\right|+(1-t)^{s}\left|f^{\prime}(b)\right|\right) d t \\
& +\int_{\frac{1}{2}}^{1} B_{t}(n+1, \alpha-n)\left(t^{s}\left|f^{\prime}(a)\right|+(1-t)^{s}\left|f^{\prime}(b)\right|\right) d t \\
& \left.-\int_{\frac{1}{2}}^{1} B_{1-t}(n+1, \alpha-n)\left(t^{s}\left|f^{\prime}(a)\right|+(1-t)^{s}\left|f^{\prime}(b)\right|\right) d t\right\}
\end{aligned}
$$




$$
\begin{aligned}
= & \frac{b-a}{2}\left\{\left|f^{\prime}(a)\right| \int_{0}^{\frac{1}{2}}\left[B_{1-t}(n+1, \alpha-n)-B_{t}(n+1, \alpha-n)\right] t^{s} d t\right. \\
& +\left|f^{\prime}(b)\right| \int_{0}^{\frac{1}{2}}\left[B_{1-t}(n+1, \alpha-n)-B_{t}(n+1, \alpha-n)\right](1-t)^{s} d t \\
& \left.+\left|f^{\prime}(a)\right| \int_{\frac{1}{2}}^{1}\left[B_{t}(n+1, \alpha-n)-B_{1-t}(n+1, \alpha-n)\right] t^{s}\right) d t \\
& +\left|f^{\prime}(b)\right| \int_{\frac{1}{2}}^{1}\left[B_{t}(n+1, \alpha-n)-B_{1-t}(n+1, \alpha-n)\right](1-t)^{s} d t .
\end{aligned}
$$

On the other hand, using the properties of incompleted beta function we have:

$$
\begin{aligned}
& B_{1-t}(n+1, \alpha-n)-B_{t}(n+1, \alpha-n) \\
= & \int_{0}^{1-t} x^{n}(1-x)^{\alpha-n-1} d x-\int_{0}^{t} x^{n}(1-x)^{\alpha-n-1} d x \\
= & \int_{t}^{1-t} x^{n}(1-x)^{\alpha-n-1} d x, \quad \text { where } 0 \leq t \leq \frac{1}{2}
\end{aligned}
$$

and

$$
\begin{aligned}
& B_{t}(n+1, \alpha-n)-B_{1-t}(n+1, \alpha-n) \\
= & \int_{0}^{t} x^{n}(1-x)^{\alpha-n-1} d x-\int_{0}^{1-t} x^{n}(1-x)^{\alpha-n-1} d x \\
= & \int_{1-t}^{t} x^{n}(1-x)^{\alpha-n-1} d x, \text { where } \frac{1}{2} \leq t \leq 1
\end{aligned}
$$

Using (3.6), (3.7) and Newton Leibnitz formula and integrating by parts we can write the following computation:

$$
\begin{aligned}
\Phi_{1}= & \int_{0}^{\frac{1}{2}}\left(\int_{t}^{1-t} x^{n}(1-x)^{\alpha-n-1} d x\right) t^{s} d t \\
= & {\left.\left[\left(\int_{t}^{1-t} x^{n}(1-x)^{\alpha-n-1} d x\right) \frac{t^{s+1}}{s+1}\right]\right|_{0} ^{\frac{1}{2}} } \\
& -\int_{0}^{\frac{1}{2}}\left(-(1-t)^{n} t^{\alpha-n-1}-t^{n}(1-t)^{\alpha-n-1}\right) \frac{t^{s+1}}{s+1} d t \\
= & \frac{1}{s+1}\left[\int_{0}^{\frac{1}{2}} t^{\alpha-n+s}(1-t)^{n} d t+\int_{0}^{\frac{1}{2}} t^{n+s+1}(1-t)^{\alpha-n-1} d t\right] \\
= & \frac{1}{s+1}\left[B_{\frac{1}{2}}(\alpha-n+s+1, n+1)+B_{\frac{1}{2}}(n+s+2, \alpha-n)\right],
\end{aligned}
$$




$$
\begin{aligned}
& \Phi_{2}=\int_{0}^{\frac{1}{2}}\left(\int_{t}^{1-t} x^{n}(1-x)^{\alpha-n-1} d x\right)(1-t)^{s} d t \\
& =\left.\left[\left(\int_{t}^{1-t} x^{n}(1-x)^{\alpha-n-1} d x\right) \frac{-(1-t)^{s+1}}{s+1}\right]\right|_{0} ^{\frac{1}{2}} \\
& -\int_{0}^{\frac{1}{2}}\left(-(1-t)^{n} t^{\alpha-n-1}-t^{n}(1-t)^{\alpha-n-1}\right) \frac{-(1-t)^{s+1}}{s+1} d t \\
& =\frac{1}{s+1} \int_{0}^{1} x^{n}(1-x)^{\alpha-n-1} d x \\
& -\frac{1}{s+1}\left[\int_{0}^{\frac{1}{2}} t^{\alpha-n-1}(1-t)^{n+s+1} d t+\int_{0}^{\frac{1}{2}} t^{n}(1-t)^{\alpha-n+s} d t\right] \\
& =\frac{1}{s+1}\left[B(n+1, \alpha-n)-B_{\frac{1}{2}}(\alpha-n, n+s+2)\right. \\
& \left.-B_{\frac{1}{2}}(n+1, \alpha-n+s+1)\right] \text {, } \\
& \Phi_{3}=\int_{\frac{1}{2}}^{1}\left(\int_{1-t}^{t} x^{n}(1-x)^{\alpha-n-1} d x\right) t^{s} d t \\
& =\left.\left[\left(\int_{1-t}^{t} x^{n}(1-x)^{\alpha-n-1} d x\right) \frac{t^{s+1}}{s+1}\right]\right|_{\frac{1}{2}} ^{1} \\
& -\frac{1}{s+1} \int_{\frac{1}{2}}^{1}\left(t^{n}(1-t)^{\alpha-n-1}+t^{\alpha-n-1}(1-t)^{n}\right) t^{s+1} d t \\
& =\frac{1}{s+1} \int_{0}^{1} x^{n}(1-x)^{\alpha-n-1} d x \\
& -\frac{1}{s+1}\left[\int_{\frac{1}{2}}^{1} t^{n+s+1}(1-t)^{\alpha-n-1} d t+\int_{\frac{1}{2}}^{1} t^{\alpha-n+s}(1-t)^{n} d t\right] \\
& =\frac{1}{s+1}\left[B(n+1, \alpha-n)-B_{\frac{1}{2}}(\alpha-n, n+s+2)\right. \\
& \left.-B_{\frac{1}{2}}(n+1, \alpha-n+s+1)\right]
\end{aligned}
$$

and

$$
\begin{aligned}
\Phi_{4}= & \int_{\frac{1}{2}}^{1}\left(\int_{1-t}^{t} x^{n}(1-x)^{\alpha-n-1} d x\right)(1-t)^{s} d t \\
= & {\left.\left[\left(\int_{1-t}^{t} x^{n}(1-x)^{\alpha-n-1} d x\right) \frac{-(1-t)^{s+1}}{s+1}\right]\right|_{\frac{1}{2}} ^{1} } \\
& +\int_{\frac{1}{2}}^{1}\left(t^{n}(1-t)^{\alpha-n-1}+t^{\alpha-n-1}(1-t)^{n}\right) \frac{(1-t)^{s+1}}{s+1} d t
\end{aligned}
$$




$$
\begin{aligned}
& =\left[\int_{\frac{1}{2}}^{1} t^{n}(1-t)^{\alpha-n+s} d t+\int_{\frac{1}{2}}^{1} t^{\alpha-n-1}(1-t)^{n+s+1} d t\right] \\
& =\frac{1}{s+1}\left[B_{\frac{1}{2}}(\alpha-n+s+1, n+1)+B_{\frac{1}{2}}(n+s+2, \alpha-n)\right],
\end{aligned}
$$

Using the fact that $B(a, b)=B_{\frac{1}{2}}(a, b)+B_{\frac{1}{2}}(b, a)$ and combining (3.8), (3.9), (3.10), (3.11) with (3.5) completes the proof.

Corollary 3.4. Taking $s=1$ in Theorem 3.3 i.e $\left|f^{\prime}\right|$ is convex, we get the following result:

$$
\begin{aligned}
& \left|B(n+1, \alpha-n)\left(\frac{f(a)+f(b)}{2}\right)-\frac{n !}{2(b-a)^{\alpha}}\left[I_{\alpha}^{a} f(b)+{ }^{b} I_{\alpha} f(a)\right]\right| \\
\leq & \frac{b-a}{2}\left(\frac{\left|f^{\prime}(a)\right|+\left|f^{\prime}(b)\right|}{2}\right) \\
& \times\left\{B_{\frac{1}{2}}(\alpha-n+2, n+1)-B_{\frac{1}{2}}(n+1, \alpha-n+2)\right. \\
& \left.+B_{\frac{1}{2}}(n+3, \alpha-n)-B_{\frac{1}{2}}(\alpha-n, n+3)+B(n+1, \alpha-n)\right\}
\end{aligned}
$$

Remark 3.5. Taking $\alpha=n+1$ in Corollary 3.4, the inequality (3.12) reduces to (1.6).

Theorem 3.6. Let $f:[a, b] \rightarrow \mathbb{R}$ be a differentiable mapping on $(a, b), a<b$ and $p>1$ with $\frac{1}{p}+\frac{1}{q}=1$. If $f^{\prime} \in L[a, b]$ and $\left|f^{\prime}\right|^{q}$ is s-convex in the second sense, then the following inequality for conformable fractional integrals holds:

$$
\begin{aligned}
& \left|B(n+1, \alpha-n)\left(\frac{f(a)+f(b)}{2}\right)-\frac{n !}{2(b-a)^{\alpha}}\left[I_{\alpha}^{a} f(b)+{ }^{b} I_{\alpha} f(a)\right]\right| \\
& \leq \frac{b-a}{2} \Psi^{\frac{1}{p}}\left[\frac{\left|f^{\prime}(a)\right|^{q}+\left|f^{\prime}(b)\right|^{q}}{s+1}\right]^{\frac{1}{q}} .
\end{aligned}
$$

where $B(a, b)$ is Euler beta function, $\alpha \in(n, n+1], n=0,1,2, \ldots$ and

$$
\Psi=2 \int_{0}^{\frac{1}{2}}\left(\int_{t}^{1-t} x^{n}(1-x)^{\alpha-n-1} d x\right)^{p}
$$


Proof. Taking modulus and using Hölder inequality with a function of $\left|f^{\prime}\right|^{q}$ convexity we get inequalities as follow:

$$
\begin{aligned}
& \left|B(n+1, \alpha-n)\left(\frac{f(a)+f(b)}{2}\right)-\frac{n !}{2(b-a)^{\alpha}}\left[I_{\alpha}^{a} f(b)+{ }^{b} I_{\alpha} f(a)\right]\right| \\
= & \frac{b-a}{2}\left|\int_{0}^{1}\left[B_{1-t}(n+1, \alpha-n)-B_{t}(n+1, \alpha-n)\right] f^{\prime}(t a+(1-t) b) d t\right| \\
\leq & \frac{b-a}{2} \int_{0}^{1}\left|B_{1-t}(n+1, \alpha-n)-B_{t}(n+1, \alpha-n)\right|\left|f^{\prime}(t a+(1-t) b)\right| d t \\
\leq & \frac{b-a}{2}\left[\int_{0}^{1}\left|B_{1-t}(n+1, \alpha-n)-B_{t}(n+1, \alpha-n)\right|^{p} d t\right]^{\frac{1}{p}} \\
& \times\left[\int_{0}^{1}\left|f^{\prime}(t a+(1-t) b)\right|^{q} d t\right]^{\frac{1}{q}} .
\end{aligned}
$$

It follows that:

$$
\begin{aligned}
\Psi= & \int_{0}^{1}\left|B_{1-t}(n+1, \alpha-n)-B_{t}(n+1, \alpha-n)\right|^{p} d t \\
= & \int_{0}^{\frac{1}{2}}\left(B_{1-t}(n+1, \alpha-n)-B_{t}(n+1, \alpha-n)\right)^{p} d t \\
& +\int_{\frac{1}{2}}^{1}\left(B_{t}(n+1, \alpha-n)-B_{1-t}(n+1, \alpha-n)\right)^{p} d t \\
= & \int_{0}^{\frac{1}{2}}\left(\int_{t}^{1-t} x^{n}(1-x)^{\alpha-n-1} d x\right)^{p} d t \\
& +\int_{\frac{1}{2}}^{1}\left(\int_{1-t}^{t} x^{n}(1-x)^{\alpha-n-1} d x\right)^{p} d t \\
= & 2 \int_{0}^{\frac{1}{2}}\left(\int_{t}^{1-t} x^{n}(1-x)^{\alpha-n-1} d x\right)^{p} d t
\end{aligned}
$$

and

$$
\begin{aligned}
& \int_{0}^{1}\left|f^{\prime}(t a+(1-t) b)\right|^{q} d t \leq\left|f^{\prime}(a)\right|^{q} \int_{0}^{1} t^{s} d t+\left|f^{\prime}(b)\right|^{q} \int_{0}^{1}(1-t)^{s} d t \\
= & \frac{1}{s+1}\left(\left|f^{\prime}(a)\right|^{q}+\left|f^{\prime}(b)\right|^{q}\right)
\end{aligned}
$$

which completes the proof.

Corollary 3.7. If we take $s=1$ in Theorem 3.6, the inequality (3.13) reduces to following inequality:

$$
\begin{aligned}
& \left|B(n+1, \alpha-n)\left(\frac{f(a)+f(b)}{2}\right)-\frac{n !}{2(b-a)^{\alpha}}\left[I_{\alpha}^{a} f(b)+{ }^{b} I_{\alpha} f(a)\right]\right| \\
\leq & \frac{b-a}{2} \Psi^{\frac{1}{p}}\left[\frac{\left|f^{\prime}(a)\right|^{q}+\left|f^{\prime}(b)\right|^{q}}{2}\right]^{\frac{1}{q}}
\end{aligned}
$$


where $B(a, b)$ is Euler beta function and

$$
\Psi=2 \int_{0}^{\frac{1}{2}}\left(\int_{t}^{1-t} x^{n}(1-x)^{\alpha-n-1} d x\right)^{p} .
$$

Corollary 3.8. If we take $\alpha=n+1$ in corollary 3.7, the inequality (3.17) reduces to following inequality:

$$
\begin{aligned}
& \left|B(\alpha, 1)\left(\frac{f(a)+f(b)}{2}\right)-\frac{\Gamma(\alpha)}{2(b-a)^{\alpha}}\left[J_{a^{+}}^{\alpha} f(b)+J_{b_{-}}^{\alpha} f(a)\right]\right| \\
\leq & \frac{b-a}{2} \Psi_{1}^{\frac{1}{p}}\left[\frac{\left|f^{\prime}(a)\right|^{q}+\left|f^{\prime}(b)\right|^{q}}{2}\right]^{\frac{1}{q}},
\end{aligned}
$$

where $\Psi_{1}=2 \int_{0}^{\frac{1}{2}}\left(\frac{(1-t)^{\alpha}-t^{\alpha}}{\alpha}\right)^{p} d t$.

Remark 3.9. If we take $\alpha=1$ in Corollary 3.8, the inequality (3.18) reduces to following inequality:

$$
\begin{aligned}
& \left|\frac{f(a)+f(b)}{2}-\frac{1}{(b-a)} \int_{a}^{b} f(x) d x\right| \\
\leq & \frac{b-a}{2}\left(\frac{1}{p+1}\right)^{\frac{1}{p}}\left[\frac{\left|f^{\prime}(a)\right|^{q}+\left|f^{\prime}(b)\right|^{q}}{2}\right]^{\frac{1}{q}},
\end{aligned}
$$

which is the same as Theorem 2.3 in [12].

Remark 3.10. If we take $\alpha \in(0,1]$ in Corollary 3.8, then the inequality (3.18) reduces to special case of Corollary 1 for $s=1$ in [19], which is the same as

$$
\begin{aligned}
& \left|\left(\frac{f(a)+f(b)}{2}\right)-\frac{\Gamma(\alpha+1)}{2(b-a)^{\alpha}}\left[J_{a^{+}}^{\alpha} f(b)+J_{b_{-}}^{\alpha} f(a)\right]\right| \\
& \leq \frac{b-a}{2}\left(\frac{1}{\alpha p+1}\right)^{\frac{1}{p}}\left[\frac{\left|f^{\prime}(a)\right|^{q}+\left|f^{\prime}(b)\right|^{q}}{2}\right]^{\frac{1}{q}} .
\end{aligned}
$$

\section{References}

[1] Abdeljawad, T., On conformable fractional calculus, J. Comput. Appl. Math., 279(2015), 57-66.

[2] Abdeljawad, T., On multiplicative fractional calculus, arxiv:1510, o4176v1 [math.CA], 6 Oct 2015.

[3] Alomari, M., Darus, M., Dragomir, S.S., Cerone, P., Ostrowski type inequalities for functions whose derivatives are s-convex in the second sense, Appl. Math. Lett., 23(9), 1071-1076.

[4] Anderson, O.R., Taylor's formula and integral inequalities for conformable fractional derivatives, arxiv:140958888v1 [math. CA], 20 Sep 2014.

[5] Avci, M., Kavurmaci, H., Özdemir, M.E., New inequalities of Hermite-Hadamard type via s-convex functions in the second sense with applications, Appl. Math. Comput., 217(12), 5171-5176. 
[6] Benkhettou, N., Hassani, S., Torres, D.E.M., A conformable fractional calculus on arbitrary time, J. King Saud Univ. Sci., 28(2016), 9398.

[7] Breckner, W.W., Stetigkeitsaussagen fr eine Klasse verallgemeinerter konvexer funktionen in topologischen linearen Raumen, Publ. Inst. Math., 23(1978), 13-20.

[8] Chen, F., Extensions of the Hermite-Hadamard Inequality for convex functions via fractional integrals, J. Math. Ineq., 10(1)(2016), 75-81.

[9] Dahmani, Z., New inequalities in fractional integrals, Int. J. Nonlinear Sci., 9(4)(2010), 493-497.

[10] Dahmani, Z., Tabharit, L., Taf, S., New generalizations of Gruss inequality using RiemannLiouville fractional integrals, Bull. Math. Anal. Appl., 2(3)(2010), 93-99.

[11] Dragomir, S.S., Agarval, R.R., The Hadamard's inequality for s-convex functions in the second sense, Demonstratio Math., 32(4)(1999), 687-696.

[12] Dragomir, S.S., Fitzpatrik, S., Two inequalities for differentiable mappings and applications to special means of real numbers and to trapezoidal formula, Appl. Math. Lett., 11(5)(1998), 91-95.

[13] Dragomir, S.S., Pearce, C.E.M., Selected Topics on Hermite-Hadamard Inequalities and Applications, RGMIA Monographs, Victoria University, 2000.

[14] Gorenflo, R., Mainardi, F., Fractional Calculus: Integral and Differential Equations of Fractional Order, 2008, arXiv preprint arXiv:0805.3823.

[15] Hudzik, H., Maligranda, L., Some remarks on s-convex functions, Aequationes Math., 48(1994), 100-111.

[16] İşcan, İ., Generalization of different type integral inequalities for s-convex functions via fractional integrals, Appl. Anal., 93(9)(2014), 1846-1862.

[17] İşcan, İ., Hermite Hadamard type inequalities for harmonically convex functions via fractional integral, Appl. Math. Comput., 238(2014), 237-244.

[18] Khalil, R., Al Horani, M., Yousef, A., Sababheh, M., A new definition of fractional derivative, J. Comput. Appl. Math., 264(2014), 65-70.

[19] Özdemir, M.E., Kavurmacı, H., Yıldız, Ç., Fractional integral inequalities via s-convex functions, Turkish J. Anal. Number Theory, 5(1)(2017), 18-22.

[20] Podlubni, I., Fractional Differential Equations, Academic Press, San Diego, 1999.

[21] Sarıkaya, M.Z., Set, E., Yaldız, H., Başak, N., Hermite-Hadamard's inequalities for fractional integrals and related fractional inequalities, Math. Comput. Model., 57(2013), 2403-2407.

[22] Set, E., Sarıkaya, M.Z., Özdemir, M.E., Yıldırım, H., The Hermite-Hadamard's inequality for some convex functions via fractional integrals and related results, J. Appl. Math. Stat. Inform., 10(2)(2014), 69-83.

[23] Set, E., New inequalities of Ostrowski type for mappings whose derivatives are s-convex in the second sense via fractional integrals, Comput. Math. Appl., 63(7)(2012), 11471154 .

[24] Set, E., Akdemir, A.O., Mumcu, İ., The Hermite-Hadamard's inequality and its extentions for conformable fractioanal integrals of any order $\alpha>0$, Submitted.

[25] Set, E., Akdemir, A.O., Mumcu, İ., Ostrowski type inequalities for functions whoose derivatives are convex via conformable fractional integrals, J. Adv. Math. Stud., 10(3)(2017), 386-395. 
[26] Set, E., Akdemir, A.O., Mumcu, İ., Chebyshev type inequalities for conformable fractional integrals, Submitted.

[27] Set, E., Mumcu, İ., Hermite-Hadamard-Fejer type inequalies for conformable fractional integrals, Submitted.

[28] Zhu, C., Feckan, M., Wang, J., Fractional integral inequalities for differentiable convex mappings and applications to special means and a midpoint formula, J. Math. Stat. Inform., 8(2)(2012), 21-28.

Erhan Set

Department of Mathematics, Faculty of Arts and Sciences, Ordu University 52200 Ordu, Turkey

e-mail: erhanset@yahoo.com

\author{
Abdurrahman Gözpinar \\ Department of Mathematics, Faculty of Arts and Sciences, Ordu University \\ 52200 Ordu, Turkey \\ e-mail: abdurrahmangozpinar79@gmail.com
}

